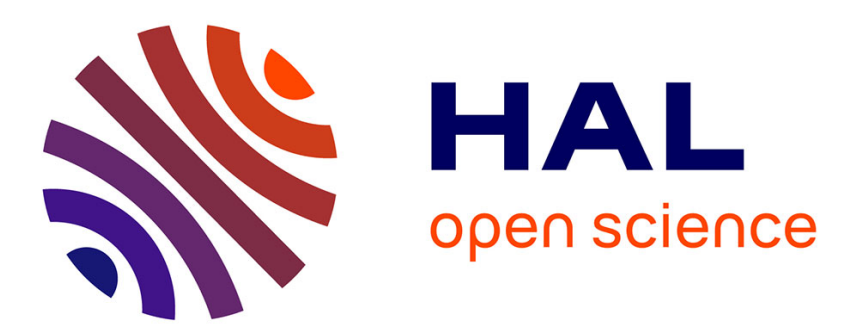

\title{
On the homogenization of a two-conductivity problem with flux jump
}

Renata Bunoiu, Claudia Timofte

\section{To cite this version:}

Renata Bunoiu, Claudia Timofte. On the homogenization of a two-conductivity problem with flux jump. Communications in Mathematical Sciences, 2017, 10.4310/CMS.2017.v15.n3.a8 . hal01528074

\section{HAL Id: hal-01528074 \\ https://hal.inria.fr/hal-01528074}

Submitted on 26 May 2017

HAL is a multi-disciplinary open access archive for the deposit and dissemination of scientific research documents, whether they are published or not. The documents may come from teaching and research institutions in France or abroad, or from public or private research centers.
L'archive ouverte pluridisciplinaire HAL, est destinée au dépôt et à la diffusion de documents scientifiques de niveau recherche, publiés ou non, émanant des établissements d'enseignement et de recherche français ou étrangers, des laboratoires publics ou privés. 


\title{
On the homogenization of a two-conductivity problem with flux jump
}

\author{
Renata BUNOIU ${ }^{1}$ and Claudia TIMOFTE ${ }^{2}$
}

\begin{abstract}
In this paper, we study the homogenization of a thermal diffusion problem in a highly heterogeneous medium formed by two constituents. The main characteristics of the medium are the discontinuity of the thermal conductivity over the domain as we go from one constituent to another and the presence of an imperfect interface between the two constituents, where both the temperature and the flux exhibit jumps. The limit problem, obtained via the periodic unfolding method, captures the influence of the jumps in the limit temperature field, in an additional source term, and in the correctors, as well.
\end{abstract}

Key words: homogenization, imperfect interface, the periodic unfolding method.

AMS subject classification: 35B27, 80M35, 80M40.

\section{Introduction}

Our goal in this paper is to analyze the effective thermal transfer in a periodic composite material formed by two constituents occupying a domain $\Omega$ in $\mathbb{R}^{N}(N \geq 2)$, divided in two open subdomains, denoted by $\Omega_{1}^{\varepsilon}$ and $\Omega_{2}^{\varepsilon}$, and separated by an imperfect interface $\Gamma^{\varepsilon}$. We assume that the phase $\Omega_{1}^{\varepsilon}$ is connected and reaches the external fixed boundary $\partial \Omega$ and that $\Omega_{2}^{\varepsilon}$ is disconnected, being the union of domains of size $\varepsilon$, periodically distributed in $\Omega$ with period of order $\varepsilon$, where $\varepsilon$ is a positive real number less than one. Nevertheless, if $N \geq 3$, our results still hold true if the domain $\Omega_{2}^{\varepsilon}$ is connected, too. The order of magnitude of the thermal conductivity of the material occupying the domain $\Omega_{2}^{\varepsilon}$ is of order $\varepsilon^{2}$, while the conductivity of the material occupying the domain $\Omega_{1}^{\varepsilon}$ is supposed to be of order one. Our problem presents various sources of singularities: the geometric one related to the interspersed periodic distribution of the components, the material one related to the conductivities and the ones generated by the presence of an imperfect interface between the two materials. All these singularities are described in terms of $\varepsilon$.

\footnotetext{
${ }^{1}$ Institut Élie Cartan de Lorraine and CNRS, UMR 7502, Université de Lorraine - Metz, France. E-mail: renata.bunoiu@univ-lorraine.fr

${ }^{2}$ University of Bucharest, Faculty of Physics, Bucharest-Magurele, P.O. Box MG-11, Romania. E-mail: claudia.timofte@g.unibuc.ro
} 
More precisely, we study the asymptotic behavior, as the small parameter $\varepsilon$ tends to zero, of the solution $u^{\varepsilon}=\left(u_{1}^{\varepsilon}, u_{2}^{\varepsilon}\right)$ of the following problem:

$$
\left\{\begin{array}{l}
-\operatorname{div}\left(A^{\varepsilon} \nabla u_{1}^{\varepsilon}\right)=f \quad \text { in } \Omega_{1}^{\varepsilon}, \\
-\operatorname{div}\left(\varepsilon^{2} A^{\varepsilon} \nabla u_{2}^{\varepsilon}\right)=f \text { in } \Omega_{2}^{\varepsilon}, \\
A^{\varepsilon} \nabla u_{1}^{\varepsilon} \cdot n^{\varepsilon}=\varepsilon h^{\varepsilon}\left(u_{1}^{\varepsilon}-u_{2}^{\varepsilon}\right)-G^{\varepsilon} \quad \text { on } \Gamma^{\varepsilon}, \\
\varepsilon^{2} A^{\varepsilon} \nabla u_{2}^{\varepsilon} \cdot n^{\varepsilon}=\varepsilon h^{\varepsilon}\left(u_{1}^{\varepsilon}-u_{2}^{\varepsilon}\right) \text { on } \Gamma^{\varepsilon}, \\
u_{1}^{\varepsilon}=0 \text { on } \partial \Omega .
\end{array}\right.
$$

The case $G^{\varepsilon}=0$, which corresponds to a continuous flux, proportional to the jump of the temperature field across the imperfect interface, has attracted, in the last two decades, the interest of a broad category of researchers. In the pioneering work [8], using the asymptotic expansion method, the authors study the homogenization of a thermal problem in a two-component composite with interfacial barrier in the particular case in which the conductivities of the two components are both of order one. For this problem, the convergence results were rigorously justified later by using various mathematical methods: the energy method in [21] and [33], the two-scale convergence method in [23] and the unfolding method for periodic homogenization in [20], [39], [40] and [32], to quote just a few of them. Also, for problems involving jumps in the solution in other contexts, such as heat transfer in polycrystals with interfacial resistance, linear elasticity problems or problems modeling the electrical conduction in biological tissues, see [4], $[5],[24],[25],[26],[30]$ and [41]. The case corresponding to the scaling of the conductivities considered in this paper is addressed, among others, in [6], [36], [35], [34], [22], [28], [1], [2]. After passing to the limit with respect to the small parameter $\varepsilon$, a regularised model of diffusion is obtained, which in fact is a special case of the double-porosity model, introduced in [37] in the frame of the heat transfer and in [9] in the context of the flow in porous media. For a review of such models in various types of fissured porous media, see, for instance, [38] and the references therein.

In this paper, we consider the case $G^{\varepsilon} \neq 0$, which corresponds to a discontinous flux as well. We study here two representative cases for the jump function $G^{\varepsilon}$, stated explicitly in Section 2, relations (2.2) and (2.3), which both lead to different modified reguralized models of diffusion. More precisely, in the first case, a new global source term, macroscopically distributed over the entire equivalent domain, appears in the right-hand side of the homogenized equation (3.8). In the second case, the novelty brought by the presence of the flux jump is the emergence of the new non homogeneous Neumann cell problem (3.25) and the presence of its solution in the corrector (3.24). We notice that this jump plays no role in the homogenized problem (3.23). Nevertheless, in Remark 3.11 we mention a case in which the homogenized problem depends on this jump, too. This last result is to be compared with the Neumann problem in perforated domains (see [14] 
and [18]), where the same cases of $G^{\varepsilon}$ are considered on the boundary of the holes and a similar phenomenon occurs. More recently, this type of functions $G^{\varepsilon}$ is encountered in [12] for the study of a thermal problem with flux jump, involving conductivities of order one and a scaling of the jump in the temperature field of order $\varepsilon^{-1}$. We also point out here the effect of the jump of the solution, which is recovered in the corrector (3.10) and in the weak limit (3.15), via the solution of the local Robin problem (3.13). This phenomenon was already noticed in the case in which $G^{\varepsilon}=0$. For transmission problems involving jump in the flux in other contexts, such as linear elasticity, theory of semiconductors, the study of photovoltaic systems, combustion theory or heat transfer problems, see [7], [10], [11], [27], [29], [31].

Let us notice that if in the microscopic problem the temperature and the flux are continuous across $\Gamma^{\varepsilon}$ and if moreover the thermal conductivities of the two materials are both of order one, we have a standard transmission problem, and, then, the limit process leads to a single-diffusion equation. However, if we assume that the thermal conductivities of the two materials are both of order one, but we keep our jump conditions at the interface, then the limit problem is a system of two coupled equations. The case $G^{\varepsilon}=0$ is studied in [23] and the limit process leads to the celebrated Barenblatt system, introduced in [9]. The case $G^{\varepsilon} \neq 0$, which leads to a modified Barenblatt model, is addressed in [13].

The rest of the paper is organized as follows: in Section 2, we introduce the microscopic problem and we fix the notation. In Section 3, we state and prove the main homogenization results of this paper. Corrector results are given, too. We end our paper with a few concluding remarks, an appendix in which we review the definition and the basic properties of the unfolding operators and their adjoints, and some references.

\section{Setting of the problem}

Let $\Omega$ be a bounded open set in $\mathbb{R}^{N}(N \geqslant 2)$, with a Lipschitz continuous boundary $\partial \Omega$ and let $Y=(0,1)^{N}$ be the reference cell in $\mathbb{R}^{N}$. We suppose that $Y_{1}$ and $Y_{2}$ are two non-empty disjoint connected open subsets of $Y$ such that $\bar{Y}_{2} \subset Y$ and $Y=Y_{1} \cup \bar{Y}_{2}$. We also assume that $\Gamma=\partial Y_{2}$ is Lipschitz continuous and that $Y_{2}$ is connected.

Throughout the paper, the small parameter $\varepsilon$ takes values in a positive real sequence tending to zero and $C$ is a positive constant independent of $\varepsilon$, whose value can change from line to line.

For each $k \in \mathbb{Z}^{N}$, we denote $Y^{k}=k+Y$ and $Y_{\alpha}^{k}=k+Y_{\alpha}$, for $\alpha=1,2$. We also define for each $\varepsilon, \mathbb{Z}_{\varepsilon}=\left\{k \in \mathbb{Z}^{N}: \quad \varepsilon \bar{Y}_{2}^{k} \subset \Omega\right\}$ and we set $\Omega_{2}^{\varepsilon}=\bigcup_{k \in \mathbb{Z}_{\varepsilon}}\left(\varepsilon Y_{2}^{k}\right)$ and $\Omega_{1}^{\varepsilon}=\Omega \backslash \bar{\Omega}_{2}^{\varepsilon}$. The boundary of $\Omega_{2}^{\varepsilon}$ is denoted by $\Gamma^{\varepsilon}$ and $n^{\varepsilon}$ is the unit outward normal to $\Omega_{2}^{\varepsilon}$.

Our goal in this paper is to analyze the asymptotic behavior, as $\varepsilon \rightarrow 0$, of the solution 
$u^{\varepsilon}=\left(u_{1}^{\varepsilon}, u_{2}^{\varepsilon}\right)$ of the following problem:

$$
\left\{\begin{array}{l}
-\operatorname{div}\left(A^{\varepsilon} \nabla u_{1}^{\varepsilon}\right)=f \quad \text { in } \Omega_{1}^{\varepsilon}, \\
-\operatorname{div}\left(\varepsilon^{2} A^{\varepsilon} \nabla u_{2}^{\varepsilon}\right)=f \text { in } \Omega_{2}^{\varepsilon}, \\
A^{\varepsilon} \nabla u_{1}^{\varepsilon} \cdot n^{\varepsilon}=\varepsilon h^{\varepsilon}\left(u_{1}^{\varepsilon}-u_{2}^{\varepsilon}\right)-G^{\varepsilon} \quad \text { on } \Gamma^{\varepsilon}, \\
\varepsilon^{2} A^{\varepsilon} \nabla u_{2}^{\varepsilon} \cdot n^{\varepsilon}=\varepsilon h^{\varepsilon}\left(u_{1}^{\varepsilon}-u_{2}^{\varepsilon}\right) \text { on } \Gamma^{\varepsilon}, \\
u_{1}^{\varepsilon}=0 \text { on } \partial \Omega .
\end{array}\right.
$$

Remark 2.1 We remark that the flux of the solution is discontinuous across $\Gamma^{\varepsilon}$. Indeed, we have

$$
A^{\varepsilon} \nabla u_{1}^{\varepsilon} \cdot n^{\varepsilon}-\varepsilon^{2} A^{\varepsilon} \nabla u_{2}^{\varepsilon} \cdot n^{\varepsilon}=-G^{\varepsilon} .
$$

The function $f \in L^{2}(\Omega)$ is given. Let $g$ be a $Y$-periodic function that belongs to $L^{2}(\Gamma)$. We define

$$
g^{\varepsilon}(x)=g\left(\frac{x}{\varepsilon}\right) \text { a.e. on } \Gamma^{\varepsilon} .
$$

For the given function $G^{\varepsilon}$ in (2.1), we consider the following two relevant situations (see, also, [12], [14] and [18]):

$$
\begin{aligned}
& \text { Case } 1: G^{\varepsilon}(x)=\varepsilon g\left(\frac{x}{\varepsilon}\right), \text { if } \mathcal{M}_{\Gamma}(g) \neq 0, \\
& \text { Case 2: } G^{\varepsilon}(x)=g\left(\frac{x}{\varepsilon}\right), \text { if } \mathcal{M}_{\Gamma}(g)=0 .
\end{aligned}
$$

Here, $\mathcal{M}_{\Gamma}(g)=\frac{1}{|\Gamma|} \int_{\Gamma} g(y) \mathrm{d} y$ denotes the mean value of the function $g$ on $\Gamma$.

Moreover, we make the following assumptions on the data:

(H1) $h$ is a $Y$-periodic function such that $h \in L^{\infty}(\Gamma)$ and there exists $h_{0} \in \mathbb{R}$ with $0<h_{0}<h(y)$ a.e. on $\Gamma$. We set

$$
h^{\varepsilon}(x)=h\left(\frac{x}{\varepsilon}\right) \quad \text { a.e. on } \Gamma^{\varepsilon} .
$$

(H2) For $\lambda, \mu \in \mathbb{R}$, with $0<\lambda \leq \mu$, let $\mathcal{M}(\lambda, \mu, Y)$ be the set of all the matrices $A \in$ $\left(L^{\infty}(Y)\right)^{N \times N}$ such that for any $\xi \in \mathbb{R}^{N}, \lambda|\xi|^{2} \leq(A(y) \xi, \xi) \leq \mu|\xi|^{2}$, almost everywhere in $Y$. For a $Y$-periodic symmetric matrix $A \in \mathcal{M}(\lambda, \mu, Y)$, we set

$$
A^{\varepsilon}(x)=A\left(\frac{x}{\varepsilon}\right) \text { a.e. in } \Omega \text {. }
$$

In order to write the variational formulation of problem (2.1), we introduce, for every positive $\varepsilon<1$, the Hilbert space

$$
H^{\varepsilon}=V^{\varepsilon} \times H^{1}\left(\Omega_{2}^{\varepsilon}\right)
$$


The space $V^{\varepsilon}=\left\{v \in H^{1}\left(\Omega_{1}^{\varepsilon}\right), v=0\right.$ on $\left.\partial \Omega\right\}$ is endowed with the norm $\|v\|_{V^{\varepsilon}}=\|\nabla v\|_{L^{2}\left(\Omega_{1}^{\varepsilon}\right)}$, for any $v \in V^{\varepsilon}$, and the space $H^{1}\left(\Omega_{2}^{\varepsilon}\right)$ is equipped with the standard norm. On the space $H^{\varepsilon}$, we consider the scalar product

$$
(u, v)_{H^{\varepsilon}}=\int_{\Omega_{1}^{\varepsilon}} \nabla u_{1} \nabla v_{1} \mathrm{~d} x+\int_{\Omega_{2}^{\varepsilon}} \varepsilon^{2} \nabla u_{2} \nabla v_{2} \mathrm{~d} x+\varepsilon \int_{\Gamma^{\varepsilon}}\left(u_{1}-u_{2}\right)\left(v_{1}-v_{2}\right) \mathrm{d} \sigma_{x}
$$

where $u=\left(u_{1}, u_{2}\right)$ and $v=\left(v_{1}, v_{2}\right)$ belong to $H^{\varepsilon}$. The norm generated by the scalar product (2.4) is given by

$$
\|v\|_{H^{\varepsilon}}^{2}=\left\|\nabla v_{1}\right\|_{L^{2}\left(\Omega_{1}^{\varepsilon}\right)}^{2}+\varepsilon^{2}\left\|\nabla v_{2}\right\|_{L^{2}\left(\Omega_{2}^{\varepsilon}\right)}^{2}+\varepsilon\left\|v_{1}-v_{2}\right\|_{L^{2}\left(\Gamma^{\varepsilon}\right)}^{2}
$$

The variational formulation of problem (2.1) is the following one: find $u^{\varepsilon} \in H^{\varepsilon}$ such that

$$
a\left(u^{\varepsilon}, v\right)=l(v), \quad \forall v \in H^{\varepsilon},
$$

where the bilinear form $a: H^{\varepsilon} \times H^{\varepsilon} \rightarrow \mathbb{R}$ and the linear form $l: H^{\varepsilon} \rightarrow \mathbb{R}$ are given by

$$
a(u, v)=\int_{\Omega_{1}^{\varepsilon}} A^{\varepsilon} \nabla u_{1} \nabla v_{1} \mathrm{~d} x+\varepsilon^{2} \int_{\Omega_{2}^{\varepsilon}} A^{\varepsilon} \nabla u_{2} \nabla v_{2} \mathrm{~d} x+\varepsilon \int_{\Gamma^{\varepsilon}} h^{\varepsilon}\left(u_{1}-u_{2}\right)\left(v_{1}-v_{2}\right) \mathrm{d} \sigma_{x}
$$

and

$$
l(v)=\int_{\Omega_{1}^{\varepsilon}} f v_{1} \mathrm{~d} x+\int_{\Omega_{2}^{\varepsilon}} f v_{2} \mathrm{~d} x+\int_{\Gamma^{\varepsilon}} G^{\varepsilon} v_{1} \mathrm{~d} \sigma_{x},
$$

respectively.

We recall in the next lemma a result from [22], which is a key argument allowing us to prove an existence and uniqueness result and a priori estimates for the solution of the variational problem (2.6). In the sequel, unless otherwise mentioned, by $C$ we denote a positive constant which is independent of $\varepsilon$ and whose value can change from line to line.

Lemma 2.2 For every $v$ given in the space $H^{\varepsilon}$, the following inequalities hold true:

$$
\left\|v_{1}\right\|_{L^{2}\left(\Omega_{1}^{\varepsilon}\right)} \leq C\|v\|_{H^{\varepsilon}}
$$

and

$$
\left\|v_{2}\right\|_{L^{2}\left(\Omega_{2}^{\varepsilon}\right)} \leq C\|v\|_{H^{\varepsilon}} .
$$

Proof. The first inequality is a direct consequence of the definition (2.5), together with the Poincaré inequality applied to functions from the space $V^{\varepsilon}$, namely

$$
\left\|v_{1}\right\|_{L^{2}\left(\Omega_{1}^{\varepsilon}\right)} \leq C\left\|\nabla v_{1}\right\|_{L^{2}\left(\Omega_{1}^{\varepsilon}\right)} .
$$

In order to prove the second inequality, we need the following inequalities (see [22]): 


$$
\left\|v_{2}\right\|_{L^{2}\left(\Omega_{2}^{\varepsilon}\right)} \leq C\left(\varepsilon\left\|\nabla v_{2}\right\|_{L^{2}\left(\Omega_{2}^{\varepsilon}\right)}+\varepsilon^{\frac{1}{2}}\left\|v_{2}\right\|_{L^{2}\left(\Gamma^{\varepsilon}\right)}\right)
$$

and

$$
\varepsilon^{\frac{1}{2}}\left\|v_{1}\right\|_{L^{2}\left(\Gamma^{\varepsilon}\right)} \leq C\left(\varepsilon\left\|\nabla v_{1}\right\|_{L^{2}\left(\Omega_{1}^{\varepsilon}\right)}+\left\|v_{1}\right\|_{L^{2}\left(\Omega_{1}^{\varepsilon}\right)}\right) .
$$

The triangular inequality applied in (2.8), together with (2.9) and (2.7), imply

$$
\begin{gathered}
\left\|v_{2}\right\|_{L^{2}\left(\Omega_{2}^{\varepsilon}\right)} \leq C\left(\varepsilon\left\|\nabla v_{2}\right\|_{L^{2}\left(\Omega_{2}^{\varepsilon}\right)}+\varepsilon^{\frac{1}{2}}\left\|v_{2}-v_{1}\right\|_{L^{2}\left(\Gamma^{\varepsilon}\right)}+\varepsilon^{\frac{1}{2}}\left\|v_{1}\right\|_{L^{2}\left(\Gamma^{\varepsilon}\right)}\right) \\
\leq C\left(\varepsilon\left\|\nabla v_{1}\right\|_{L^{2}\left(\Omega_{1}^{\varepsilon}\right)}+\varepsilon\left\|\nabla v_{2}\right\|_{L^{2}\left(\Omega_{2}^{\varepsilon}\right)}+\varepsilon^{\frac{1}{2}}\left\|v_{1}-v_{2}\right\|_{L^{2}\left(\Gamma^{\varepsilon}\right)}+\left\|v_{1}\right\|_{L^{2}\left(\Omega_{1}^{\varepsilon}\right)}\right) \\
\leq C\left(\left\|\nabla v_{1}\right\|_{L^{2}\left(\Omega_{1}^{\varepsilon}\right)}+\varepsilon\left\|\nabla v_{2}\right\|_{L^{2}\left(\Omega_{2}^{\varepsilon}\right)}+\varepsilon^{\frac{1}{2}}\left\|v_{1}-v_{2}\right\|_{L^{2}\left(\Gamma^{\varepsilon}\right)}\right),
\end{gathered}
$$

and the second inequality then follows, by using the definition (2.5).

Theorem 2.3 For any $\varepsilon \in(0,1)$, the variational problem (2.6) has a unique solution $u^{\varepsilon} \in H^{\varepsilon}$. Moreover, there exists a constant $C>0$, independent of $\varepsilon$, such that

$$
\left\|u_{1}^{\varepsilon}\right\|_{L^{2}\left(\Omega_{1}^{\varepsilon}\right)} \leq C, \quad\left\|u_{2}^{\varepsilon}\right\|_{L^{2}\left(\Omega_{2}^{\varepsilon}\right)} \leq C
$$

and

$$
\left\|\nabla u_{1}^{\varepsilon}\right\|_{L^{2}\left(\Omega_{1}^{\varepsilon}\right)} \leq C, \quad \varepsilon\left\|\nabla u_{2}^{\varepsilon}\right\|_{L^{2}\left(\Omega_{2}^{\varepsilon}\right)} \leq C, \quad \varepsilon^{1 / 2}\left\|u_{1}^{\varepsilon}-u_{2}^{\varepsilon}\right\|_{L^{2}\left(\Gamma^{\varepsilon}\right)} \leq C .
$$

Proof. In order to prove the existence and the uniqueness of the solution for problem (2.6), we apply the Lax-Milgram theorem for the space $H^{\varepsilon}$ endowed with the norm (2.5). Due to the hypotheses $(H 1)$ and $(H 2)$, we easily get that the bilinear form $a$ is coercive and continuous. Indeed, we have

$$
a(v, v) \geq C\|v\|_{H^{\varepsilon}}^{2}, \quad \forall v \in H^{\varepsilon},
$$

and

$$
a(u, v) \leq C\|u\|_{H^{\varepsilon}}\|v\|_{H^{\varepsilon}}, \quad \forall u, v \in H^{\varepsilon} .
$$

Let us prove now that the linear form $l$ is continuous, i.e.

$$
l(v) \leq C\|v\|_{H^{\varepsilon}}, \quad \forall v \in H^{\varepsilon} .
$$

One obviously has

$$
|l(v)| \leq\|f\|_{L^{2}\left(\Omega_{1}^{\varepsilon}\right)}\left\|v_{1}\right\|_{L^{2}\left(\Omega_{1}^{\varepsilon}\right)}+\|f\|_{L^{2}\left(\Omega_{1}^{\varepsilon}\right)}\left\|v_{2}\right\|_{L^{2}\left(\Omega_{2}^{\varepsilon}\right)}+\left|\int_{\Gamma^{\varepsilon}} G^{\varepsilon}(x) v_{1}(x) \mathrm{d} \sigma_{x}\right| .
$$

According to Proposition 3.8 in [18], we obtain the estimate of the last term in (2.12) as follows:

(i) if $G^{\varepsilon}$ satisfies $(2.2)$, then

$$
\left|\int_{\Gamma^{\varepsilon}} G^{\varepsilon}(x) v_{1}(x) \mathrm{d} \sigma_{x}\right|=\left|\int_{\Gamma^{\varepsilon}} \varepsilon g\left(\frac{x}{\varepsilon}\right) v_{1}(x) \mathrm{d} \sigma_{x}\right| \leq \varepsilon \frac{C}{\varepsilon}\left(\left|\mathcal{M}_{\Gamma}(g)\right|+\varepsilon\right)\left\|\nabla v_{1}\right\|_{L^{2}\left(\Omega_{1}^{\varepsilon}\right)} \leq C\left\|\nabla v_{1}\right\|_{L^{2}\left(\Omega_{1}^{\varepsilon}\right)} ;
$$


(ii) if $G^{\varepsilon}$ satisfies (2.3), then

$\left|\int_{\Gamma^{\varepsilon}} G^{\varepsilon}(x) v_{1}(x) \mathrm{d} \sigma_{x}\right|=\left|\int_{\Gamma^{\varepsilon}} g\left(\frac{x}{\varepsilon}\right) v_{1}(x) \mathrm{d} \sigma_{x}\right| \leq \frac{C}{\varepsilon}\left(\left|\mathcal{M}_{\Gamma}(g)\right|+\varepsilon\right)\left\|\nabla v_{1}\right\|_{L^{2}\left(\Omega_{1}^{\varepsilon}\right)} \leq C\left\|\nabla v_{1}\right\|_{L^{2}\left(\Omega_{1}^{\varepsilon}\right)}$, since $\mathcal{M}_{\Gamma}(g)=0$.

Coming back to (2.12), we obtain:

$$
|l(v)| \leq C\left(\left\|v_{1}\right\|_{L^{2}\left(\Omega_{1}^{\varepsilon}\right)}+\left\|v_{2}\right\|_{L^{2}\left(\Omega_{2}^{\varepsilon}\right)}+\left\|\nabla v_{1}\right\|_{L^{2}\left(\Omega_{1}^{\varepsilon}\right)}\right) .
$$

By using Lemma 2.2 and the definition (2.5), we get the continuity of $l$. Thus, the Lax-Milgram theorem applies.

In order to obtain the a priori estimates (2.10) and (2.11), we take $v=u^{\varepsilon}$ in the variational formulation (2.6). By using the coerciveness of $a$ and the continuity of $l$, we obtain

$$
\left\|u^{\varepsilon}\right\|_{H^{\varepsilon}} \leq C
$$

which obviously imply (2.11). Estimates (2.10) are then obtained by applying Lemma 2.2.

\section{Homogenization results}

Our goal in this section is to pass to the limit, with $\varepsilon \rightarrow 0$, in the variational formulation (2.6) of the problem (2.1). To this end, we make use of the periodic unfolding method and the general compactness results given in the appendix of this paper.

More precisely, using the a priori estimates (2.10)-(2.11) and the general compactness results from Proposition 5.6, it follows that there exist $u_{1} \in H_{0}^{1}(\Omega), \widehat{u}_{1} \in L^{2}\left(\Omega, H_{\text {per }}^{1}\left(Y_{1}\right)\right)$, $\widehat{u}_{2} \in L^{2}\left(\Omega, H^{1}\left(Y_{2}\right)\right)$ such that $\mathcal{M}_{\Gamma}\left(\widehat{u}_{1}\right)=0$ and up to a subsequence, for $\varepsilon \rightarrow 0$, we get:

$$
\begin{aligned}
& \mathcal{T}_{1}^{\varepsilon}\left(u_{1}^{\varepsilon}\right) \rightarrow u_{1} \quad \text { strongly in } L^{2}\left(\Omega, H^{1}\left(Y_{1}\right)\right), \\
& \mathcal{T}_{1}^{\varepsilon}\left(\nabla u_{1}^{\varepsilon}\right) \rightarrow \nabla u_{1}+\nabla_{y} \widehat{u}_{1} \quad \text { weakly in } L^{2}\left(\Omega \times Y_{1}\right), \\
& \mathcal{T}_{2}^{\varepsilon}\left(u_{2}^{\varepsilon}\right) \rightarrow \widehat{u}_{2} \quad \text { weakly in } L^{2}\left(\Omega, H^{1}\left(Y_{2}\right)\right), \\
& \varepsilon \mathcal{T}_{2}^{\varepsilon}\left(\nabla u_{2}^{\varepsilon}\right) \rightarrow \nabla_{y} \widehat{u}_{2} \quad \text { weakly in } L^{2}\left(\Omega \times Y_{2}\right), \\
& \widetilde{u}_{1}^{\varepsilon} \rightarrow\left|Y_{1}\right| u_{1} \quad \text { weakly in } L^{2}(\Omega), \\
& \widetilde{u}_{2}^{\varepsilon} \rightarrow \int_{Y_{2}} \widehat{u}_{2}(x, y) \mathrm{d} y \quad \text { weakly in } L^{2}(\Omega),
\end{aligned}
$$

where the space $H_{\mathrm{per}}^{1}\left(Y_{1}\right)$ is defined by

$$
H_{\text {per }}^{1}\left(Y_{1}\right)=\left\{v \in H^{1}\left(Y_{1}\right) \mid v \text { is Y-periodic }\right\} .
$$

Remark 3.1 We notice that in (3.1) we omitted to write $|Y|$. Indeed, since $Y$ is the unit cube, we have $|Y|=1$ and, in order to simplify the presentation, in the sequel we shall not write it. 
Let $W_{\text {per }}\left(Y_{1}\right)=\left\{v \in H_{\text {per }}^{1}\left(Y_{1}\right) \mid \mathcal{M}_{\Gamma}(v)=0\right\}$. We introduce the space

$$
\mathcal{V}=H_{0}^{1}(\Omega) \times L^{2}\left(\Omega ; W_{\mathrm{per}}\left(Y_{1}\right)\right) \times L^{2}\left(\Omega, H^{1}\left(Y_{2}\right)\right)
$$

equipped with the norm

$$
\|V\|_{\mathcal{V}}^{2}=\left\|\nabla v+\nabla_{y} \widehat{v}_{1}\right\|_{L^{2}\left(\Omega \times Y_{1}\right)}^{2}+\left\|\nabla_{y} \widehat{v}_{2}\right\|_{L^{2}\left(\Omega \times Y_{2}\right)}^{2}+\left\|v-\widehat{v}_{2}\right\|_{L^{2}(\Omega \times \Gamma)}^{2},
$$

for all $V=\left(v, \widehat{v}_{1}, \widehat{v}_{2}\right) \in \mathcal{V}$.

In order to pass to the limit in (2.6), we need to distinguish between two cases, depending on the form of the function $G^{\varepsilon}$.

Case 1: $G^{\varepsilon}=\varepsilon g\left(\frac{x}{\varepsilon}\right)$, if $\mathcal{M}_{\Gamma}(g) \neq 0$.

Theorem 3.2 The unique solution $u^{\varepsilon}=\left(u_{1}^{\varepsilon}, u_{2}^{\varepsilon}\right)$ of the variational problem (2.6) converges, in the sense of (3.1), to the unique solution $\left(u_{1}, \widehat{u}_{1}, \widehat{u}_{2}\right) \in \mathcal{V}$ of the following unfolded limit problem:

$$
\begin{gathered}
\int_{\Omega \times Y_{1}} A(y)\left(\nabla u_{1}+\nabla_{y} \widehat{u}_{1}\right)\left(\nabla \varphi+\nabla_{y} \Phi_{1}\right) \mathrm{d} x \mathrm{~d} y+\int_{\Omega \times Y_{2}} A(y) \nabla_{y} \widehat{u}_{2} \nabla_{y} \Phi_{2} \mathrm{~d} x \mathrm{~d} y+ \\
\int_{\Omega \times \Gamma} h(y)\left(u_{1}-\widehat{u}_{2}\right)\left(\varphi-\Phi_{2}\right) \mathrm{d} x \mathrm{~d} \sigma_{y}=\int_{\Omega \times Y_{1}} f(x) \varphi(x) \mathrm{d} x \mathrm{~d} y+\int_{\Omega \times Y_{2}} f(x) \Phi_{2}(x, y) \mathrm{d} x \mathrm{~d} y+ \\
|\Gamma| \mathcal{M}_{\Gamma}(g) \int_{\Omega} \varphi(x) \mathrm{d} x,
\end{gathered}
$$

for all $\varphi \in H_{0}^{1}(\Omega), \Phi_{1} \in L^{2}\left(\Omega, H_{p e r}^{1}\left(Y_{1}\right)\right)$ and $\Phi_{2} \in L^{2}\left(\Omega, H^{1}\left(Y_{2}\right)\right)$.

Proof. In order to get the limit problem (3.2), we unfold the variational formulation (2.6). Then, by using Proposition 5.3 and Lemma 5.4, we obtain

$$
\begin{gathered}
\lim _{\varepsilon \rightarrow 0}\left(\int_{\Omega \times Y_{1}} \mathcal{T}_{1}^{\varepsilon}\left(A^{\varepsilon}\right) \mathcal{T}_{1}^{\varepsilon}\left(\nabla u_{1}^{\varepsilon}\right) \mathcal{T}_{1}^{\varepsilon}\left(\nabla v_{1}\right) \mathrm{d} x+\int_{\Omega \times Y_{2}} \mathcal{T}_{2}^{\varepsilon}\left(A^{\varepsilon}\right) \mathcal{T}_{2}^{\varepsilon}\left(\varepsilon \nabla u_{2}^{\varepsilon}\right) \mathcal{T}_{2}^{\varepsilon}\left(\varepsilon \nabla v_{2}\right) \mathrm{d} x+\right. \\
\left.\int_{\Omega \times \Gamma} h(y)\left(\mathcal{T}_{1}^{\varepsilon}\left(u_{1}^{\varepsilon}\right)-\mathcal{T}_{2}^{\varepsilon}\left(u_{2}^{\varepsilon}\right)\right)\left(\mathcal{T}_{1}^{\varepsilon}\left(v_{1}\right)-\mathcal{T}_{2}^{\varepsilon}\left(v_{2}\right)\right) \mathrm{d} \sigma_{x}\right)= \\
\lim _{\varepsilon \rightarrow 0}\left(\int_{\Omega \times Y_{1}} \mathcal{T}_{1}^{\varepsilon}(f) \mathcal{T}_{1}^{\varepsilon}\left(v_{1}\right) \mathrm{d} x+\int_{\Omega \times Y_{2}} \mathcal{T}_{2}^{\varepsilon}(f) \mathcal{T}_{2}^{\varepsilon}\left(v_{2}\right) \mathrm{d} x+\frac{1}{\varepsilon} \int_{\Omega \times \Gamma} \mathcal{T}_{b}^{\varepsilon}\left(G^{\varepsilon}\right) \mathcal{T}_{b}^{\varepsilon}\left(v_{1}\right) \mathrm{d} \sigma_{x}\right) .
\end{gathered}
$$

In this unfolded problem, we choose the admissible test functions

$$
v_{1}=\varphi(x)+\varepsilon \omega_{1}(x) \psi_{1}\left(\frac{x}{\varepsilon}\right), \quad v_{2}=\omega_{2}(x) \psi_{2}\left(\frac{x}{\varepsilon}\right),
$$

with $\varphi, \omega_{1}, \omega_{2} \in \mathcal{D}(\Omega), \psi_{1} \in H_{\text {per }}^{1}\left(Y_{1}\right), \psi_{2} \in H^{1}\left(Y_{2}\right)$. It is not difficult to see that we have the following convergences:

$$
\mathcal{T}_{1}^{\varepsilon}\left(v_{1}\right) \rightarrow \varphi(x) \text { strongly in } L^{2}\left(\Omega \times Y_{1}\right),
$$




$$
\begin{gathered}
\mathcal{T}_{1}^{\varepsilon}\left(\nabla v_{1}\right) \rightarrow \nabla \varphi(x)+\nabla_{y} \Phi_{1} \quad \text { strongly in } L^{2}\left(\Omega \times Y_{1}\right), \\
\mathcal{T}_{2}^{\varepsilon}\left(v_{2}\right) \rightarrow \Phi_{2}(x, y) \quad \text { strongly in } L^{2}\left(\Omega \times Y_{2}\right),
\end{gathered}
$$

and

$$
\mathcal{T}_{2}^{\varepsilon}\left(\varepsilon \nabla v_{2}\right) \rightarrow \nabla_{y} \Phi_{2} \quad \text { strongly in } L^{2}\left(\Omega \times Y_{2}\right),
$$

where $\Phi_{1}(x, y)=\omega_{1}(x) \psi_{1}(y)$ and $\Phi_{2}(x, y)=\omega_{2}(x) \psi_{2}(y)$.

The passage to the limit with $\varepsilon \rightarrow 0$ is standard, by using convergences (3.1) and (3.4)-(3.7). The only term which needs more attention is the one involving the function $G^{\varepsilon}$. For this term, we get:

$$
\begin{gathered}
\frac{1}{\varepsilon} \int_{\Omega \times \Gamma} \mathcal{T}_{b}^{\varepsilon}\left(G^{\varepsilon}\right) \mathcal{T}_{b}^{\varepsilon}\left(v_{1}\right) \mathrm{d} \sigma_{x}=\int_{\Omega \times \Gamma} \mathcal{T}_{b}^{\varepsilon}\left(g\left(\frac{x}{\varepsilon}\right)\right) \mathcal{T}_{b}^{\varepsilon}\left(\varphi(x)+\varepsilon \omega_{1}(x) \psi_{1}\left(\frac{x}{\varepsilon}\right)\right) \mathrm{d} \sigma_{x}= \\
\int_{\Omega \times \Gamma} g(y) \mathcal{T}_{b}^{\varepsilon}(\varphi)(x, y) \mathrm{d} x \mathrm{~d} \sigma_{y}+\varepsilon \int_{\Omega \times \Gamma} g(y) \mathcal{T}_{b}^{\varepsilon}\left(\omega_{1}\right)(x, y) \mathcal{T}_{b}^{\varepsilon}\left(\psi_{1}\right)(x, y) \mathrm{d} x \mathrm{~d} \sigma_{y} \rightarrow \\
|\Gamma| \mathcal{M}_{\Gamma}(g) \int_{\Omega} \varphi(x) \mathrm{d} x .
\end{gathered}
$$

By the density of $\mathcal{D}(\Omega) \otimes H_{\text {per }}^{1}\left(Y_{1}\right)$ in $L^{2}\left(\Omega, H_{\text {per }}^{1}\left(Y_{1}\right)\right)$ and of $\mathcal{D}(\Omega) \otimes H^{1}\left(Y_{2}\right)$ in $L^{2}\left(\Omega, H^{1}\left(Y_{2}\right)\right)$, we obtain (3.2).

We notice that our limit problem (3.2) is similar with the one obtained in [34], the only difference being the right-hand side, in which an extra constant term involving the function $g$ arises. Indeed, our right-hand side actually writes

$$
\int_{\Omega} F(x) \varphi(x) \mathrm{d} x+\int_{\Omega \times Y_{2}} f(x) \Phi_{2}(x, y) \mathrm{d} x \mathrm{~d} y,
$$

with

$$
F(x)=\left|Y_{1}\right| f(x)+|\Gamma| \mathcal{M}_{\Gamma}(g) .
$$

The existence and the uniqueness for the solution of problem (3.2) is a consequence of the Lax-Milgram theorem. Due to the uniqueness of $\left(u_{1}, \widehat{u}_{1}, \widehat{u}_{2}\right) \in \mathcal{V}$, all the above convergences hold true for the whole sequence.

Theorem 3.3 The unique solution $u^{\varepsilon}=\left(u_{1}^{\varepsilon}, u_{2}^{\varepsilon}\right)$ of the variational problem (2.6) converges, in the sense of (3.1), to $\left(u_{1}, \widehat{u}_{1}, \widehat{u}_{2}\right) \in \mathcal{V}$, where $u_{1}$ is the unique solution of the homogenized problem

$$
\left\{\begin{array}{l}
-\operatorname{div}\left(A^{h o m} \nabla u_{1}(x)\right)=f(x)+|\Gamma| \mathcal{M}_{\Gamma}(g) \quad \text { in } \Omega \\
u_{1}=0 \quad \text { on } \partial \Omega
\end{array}\right.
$$

and

$$
\widehat{u}_{1}(x, y)=-\sum_{j=1}^{N} \frac{\partial u_{1}}{\partial x_{j}}(x) \chi_{1}^{j}(y) \quad \text { in } \Omega \times Y_{1},
$$




$$
\widehat{u}_{2}(x, y)=u_{1}(x)+f(x) \chi_{2}(y) \quad \text { in } \Omega \times Y_{2} .
$$

Here, $A^{\text {hom }}$ is the constant homogenized matrix whose entries are defined, for $i, j=1, \ldots, N$ by

$$
A_{i j}^{h o m}=\int_{Y_{1}}\left(a_{i j}-\sum_{k=1}^{N} a_{i k} \frac{\partial \chi_{1}^{j}}{\partial y_{k}}\right) \mathrm{d} y .
$$

The vectorial function $\chi_{1}^{j} \in H_{p e r}^{1}\left(Y_{1}\right)(j=1, \ldots, N)$ and the scalar function $\chi_{2} \in H^{1}\left(Y_{2}\right)$ are the weak solutions of the following cell problems:

$$
\left\{\begin{array}{lc}
-\operatorname{div}_{y}\left(A(y)\left(\nabla_{y} \chi_{1}^{j}-e_{j}\right)\right)=0 & \text { in } Y_{1} \\
\left(A(y)\left(\nabla_{y} \chi_{1}^{j}-e_{j}\right)\right) \cdot n=0 & \text { on } \Gamma \\
\mathcal{M}_{\Gamma}\left(\chi_{1}^{j}\right)=0 &
\end{array}\right.
$$

and

$$
\left\{\begin{array}{l}
-\operatorname{div}_{y}\left(A(y) \nabla_{y} \chi_{2}\right)=1 \quad \text { in } Y_{2}, \\
A(y) \nabla_{y} \chi_{2} \cdot n+h \chi_{2}=0 \quad \text { on } \Gamma
\end{array}\right.
$$

where $n$ denotes the unit outward normal to $Y_{2}$.

Moreover, the weak solution $\left(u_{1}^{\varepsilon}, u_{2}^{\varepsilon}\right)$ of problem (2.6) verifies:

$$
\widetilde{u}_{1}^{\varepsilon} \rightarrow\left|Y_{1}\right| u_{1} \quad \text { weakly in } L^{2}(\Omega)
$$

and

$$
\widetilde{u}_{2}^{\varepsilon} \rightarrow\left|Y_{2}\right| u_{1}+\left(\int_{Y_{2}} \chi_{2}(y) \mathrm{d} y\right) f \text { weakly in } L^{2}(\Omega) .
$$

Proof. By choosing $\varphi=0$ in the unfolded limit problem (3.2), we obtain:

$$
\begin{gathered}
\int_{\Omega \times Y_{1}} A(y)\left(\nabla u_{1}+\nabla_{y} \widehat{u}_{1}\right) \nabla_{y} \Phi_{1} \mathrm{~d} x \mathrm{~d} y+\int_{\Omega \times Y_{2}} A(y)\left(\nabla_{y} \widehat{u}_{2}\right) \nabla_{y} \Phi_{2} \mathrm{~d} x \mathrm{~d} y- \\
\int_{\Omega \times \Gamma} h(y)\left(u_{1}-\widehat{u}_{2}\right) \Phi_{2} \mathrm{~d} x \mathrm{~d} \sigma_{y}=\int_{\Omega \times Y_{2}} f \Phi_{2} \mathrm{~d} x \mathrm{~d} y .
\end{gathered}
$$

Then, taking $\Phi_{2}=0$ in (3.16), we get:

$$
\int_{\Omega \times Y_{1}} A(y)\left(\nabla u_{1}+\nabla_{y} \widehat{u}_{1}\right) \nabla_{y} \Phi_{1} \mathrm{~d} x \mathrm{~d} y=0
$$

which implies

$$
-\operatorname{div}_{y}\left(A(y) \nabla_{y} \widehat{u}_{1}\right)=\operatorname{div}_{y}\left(A(y) \nabla u_{1}\right) \quad \text { in } \Omega \times Y_{1}
$$

and

$$
A(y)\left(\nabla u_{1}+\nabla_{y} \widehat{u}_{1}\right) \cdot n=0 \quad \text { on } \Omega \times \Gamma .
$$

Classical results from the theory of homogenization then imply (3.9) and (3.12). 
By choosing now $\Phi_{1}=0$ in (3.16), we get:

$$
\int_{\Omega \times Y_{2}} A(y)\left(\nabla_{y} \widehat{u}_{2}\right) \nabla_{y} \Phi_{2} \mathrm{~d} x \mathrm{~d} y-\int_{\Omega \times \Gamma} h(y)\left(u_{1}-\widehat{u}_{2}\right) \Phi_{2} \mathrm{~d} x \mathrm{~d} \sigma_{y}=\int_{\Omega \times Y_{2}} f \Phi_{2} \mathrm{~d} x \mathrm{~d} y,
$$

which implies

$$
-\operatorname{div}_{y}\left(A(y) \nabla_{y} \widehat{u}_{2}\right)=f \quad \text { in } \Omega \times Y_{2}
$$

and

$$
A(y) \nabla_{y} \widehat{u}_{2} \cdot n=h(y)\left(u_{1}-\widehat{u}_{2}\right) \quad \text { on } \Omega \times \Gamma .
$$

This suggests us to search the function $\widehat{u}_{2}$ of the form

$$
\widehat{u}_{2}(x, y)=u_{1}(x)+f(x) \chi_{2}(y) \quad \text { in } \Omega \times Y_{2} .
$$

By replacing this form of $\widehat{u}_{2}$ in the two previous equations, we obtain

$$
-\operatorname{div}_{y}\left(A(y)\left(f(x) \nabla_{y} \chi_{2}\right)\right)=f(x) \quad \text { in } \Omega \times Y_{2},
$$

and

$$
A(y)\left(f(x) \nabla_{y} \chi_{2}\right) \cdot n=-h(y) f(x) \chi_{2}(y) \quad \text { on } \Omega \times \Gamma,
$$

which imply that the scalar function $\chi_{2}$ is the solution of the Robin cell problem (3.13).

By choosing now $\Phi_{1}=\Phi_{2}=0$ in (3.2), we obtain:

We have the equality

$$
\begin{gathered}
\int_{\Omega \times Y_{1}} A(y)\left(\nabla u_{1}+\nabla_{y} \widehat{u}_{1}\right) \nabla \varphi \mathrm{d} x \mathrm{~d} y+\int_{\Omega \times \Gamma} h(y)\left(u_{1}-\widehat{u}_{2}\right) \varphi \mathrm{d} x \mathrm{~d} \sigma_{y}= \\
\left|Y_{1}\right| \int_{\Omega} f(x) \varphi(x) \mathrm{d} x+|\Gamma| \mathcal{M}_{\Gamma}(g) \int_{\Omega} \varphi(x) \mathrm{d} x .
\end{gathered}
$$

$$
\begin{gathered}
\int_{\Omega \times \Gamma} h(y)\left(u_{1}-\widehat{u}_{2}\right) \varphi \mathrm{d} x \mathrm{~d} \sigma_{y}=-\int_{\Omega \times \Gamma} h(y) f(x) \chi_{2}(y) \varphi(x) \mathrm{d} x \mathrm{~d} \sigma_{y}= \\
\left(-\int_{\Gamma} h(y) \chi_{2}(y) \mathrm{d} \sigma_{y}\right) \int_{\Omega} f(x) \varphi(x) \mathrm{d} x=\left(\int_{\Gamma} A(y) \nabla_{y} \chi_{2}(y) \cdot n \mathrm{~d} \sigma_{y}\right) \int_{\Omega} f(x) \varphi(x) \mathrm{d} x= \\
\left(\int_{Y_{2}} \operatorname{div}_{y}\left(A(y) \nabla_{y} \chi_{2}(y)\right) \mathrm{d} y\right) \int_{\Omega} f(x) \varphi(x) \mathrm{d} x= \\
\int_{Y_{2}}(-1) \mathrm{d} y \int_{\Omega} f(x) \varphi(x) \mathrm{d} x=-\left|Y_{2}\right| \int_{\Omega} f(x) \varphi(x) \mathrm{d} x
\end{gathered}
$$

and then relation (3.17) becomes:

$$
\int_{\Omega \times Y_{1}} A(y)\left(\nabla u_{1}+\nabla_{y} \widehat{u}_{1}\right) \nabla \varphi \mathrm{d} x \mathrm{~d} y=\int_{\Omega} f(x) \varphi(x) \mathrm{d} x+|\Gamma| \mathcal{M}_{\Gamma}(g) \int_{\Omega} \varphi(x) \mathrm{d} x .
$$

We integrate this last equality by parts with respect to $x$ and, by using (3.9) and (3.12), we are led to the homogenized problem (3.8). 
Remark 3.4 Due to the right scaling $\varepsilon$ in front of the function $g^{\varepsilon}$ given at the interface $\Gamma^{\varepsilon}$, we obtain at the limit a new source term distributed all over the domain $\Omega$. Our initial problem (2.1) can be also studied for a nonzero function $g$ with mean-value $\mathcal{M}_{\Gamma}(g)$ equal to zero. In this situation, there is no contribution of $g$ in the right-hand side of the homogenized equation and, thus, the limit problem is the same as in the case with no $g$ at all in the microscopic problem.

Remark 3.5 The solution $u_{1}(x)$ of problem (3.8) represents the contribution coming from the first material distributed in $\Omega_{1}^{\varepsilon}$ and the solution $\widehat{u}_{2}(x, y)$, verifying relation (3.10), is an additional contribution coming from the second material distributed in $\Omega_{2}^{\varepsilon}$. This shows that the diffusion within the domain $\Omega \times Y_{2}$ has more than a local character. The limit problems keep information from the two different materials, but on two different scales, and this is an important particularity of such models.(see e.g. [3]) We remark that the homogenized matrix $A^{\text {hom }}$ and, so, the solution $u_{1}$, are independent of the function $h$, while the limit $\widehat{u}_{2}$ depends on $h$, via the function $\chi_{2}$.

Remark 3.6 All the above results remain true for the case in which the set $Y_{2}$ is not connected, but consists on a finite number of connected components, as in [20].

We can also state the convergence of the energy and corrector results for the solution $u^{\varepsilon}=$ $\left(u_{1}^{\varepsilon}, u_{2}^{\varepsilon}\right)$ of problem (2.1). They are obtained in a classical way, by adapting to our case the proof of Proposition 4.7 in [20]. We have the following result:

Theorem 3.7 Under the assumptions of Theorem 3.1, if $u^{\varepsilon}=\left(u_{1}^{\varepsilon}, u_{2}^{\varepsilon}\right)$ is the unique solution of problem (2.1), then

$$
\begin{gathered}
\lim _{\varepsilon \rightarrow 0}\left(\int_{\Omega_{1}^{\varepsilon}} A^{\varepsilon} \nabla u_{1}^{\varepsilon} \nabla u_{1}^{\varepsilon} \mathrm{d} x+\int_{\Omega_{2}^{\varepsilon}} \varepsilon^{2} A^{\varepsilon} \nabla u_{2}^{\varepsilon} \nabla u_{2}^{\varepsilon} \mathrm{d} x\right)= \\
\int_{\Omega \times Y_{1}} A(y)\left(\nabla u_{1}+\nabla_{y} \widehat{u}_{1}\right)\left(\nabla u_{1}+\nabla_{y} \widehat{u}_{1}\right) \mathrm{d} x \mathrm{~d} y+ \\
\int_{\Omega \times Y_{2}} A(y) \nabla_{y} \widehat{u}_{2} \nabla_{y} \widehat{u}_{2} \mathrm{~d} x \mathrm{~d} y, \\
\lim _{\varepsilon \rightarrow 0}\left(\int_{\Lambda_{1}^{\varepsilon}}\left|\nabla u_{1}^{\varepsilon}\right|^{2} \mathrm{~d} x+\int_{\Lambda_{2}^{\varepsilon}}\left|\nabla u_{2}^{\varepsilon}\right|^{2} \mathrm{~d} x\right)=0, \\
\mathcal{T}_{1}^{\varepsilon}\left(\nabla u_{1}^{\varepsilon}\right) \rightarrow \nabla u_{1}+\nabla_{y} \widehat{u}_{1} \quad \text { strongly in } L^{2}\left(\Omega \times Y_{1}\right)
\end{gathered}
$$

and

$$
\mathcal{T}_{2}^{\varepsilon}\left(\varepsilon \nabla u_{2}^{\varepsilon}\right) \rightarrow \nabla_{y} \widehat{u}_{2} \quad \text { strongly in } L^{2}\left(\Omega \times Y_{2}\right)
$$


Moreover, the following corrector result holds true:

$$
\left\|\nabla u_{1}^{\varepsilon}-\nabla u_{1}+\sum_{j=1}^{N} \mathcal{U}_{1}^{\varepsilon}\left(\frac{\partial u_{1}}{\partial x_{j}}\right) \mathcal{U}_{1}^{\varepsilon}\left(\nabla_{y} \chi_{1}^{j}\right)\right\|_{L^{2}\left(\Omega_{1}^{\varepsilon}\right)} \longrightarrow 0
$$

and

$$
\left\|\nabla u_{2}^{\varepsilon}-\nabla u_{1}-f(x) \mathcal{U}_{2}^{\varepsilon}\left(\nabla_{y} \chi_{2}\right)\right\|_{L^{2}\left(\Omega_{2}^{\varepsilon}\right)} \longrightarrow 0
$$

Let us analyse now the second relevant situation for the jump function $G^{\varepsilon}$.

Case 2: $G^{\varepsilon}(x)=g\left(\frac{x}{\varepsilon}\right)$, if $\mathcal{M}_{\Gamma}(g)=0$.

Theorem 3.8 The unique solution $u^{\varepsilon}=\left(u_{1}^{\varepsilon}, u_{2}^{\varepsilon}\right)$ of the variational problem (2.6) converges, in the sense of (3.1), to the unique solution $\left(u_{1}, \widehat{u}_{1}, \widehat{u}_{2}\right) \in \mathcal{V}$ of the following unfolded limit problem:

$$
\begin{gathered}
\int_{\Omega \times Y_{1}} A(y)\left(\nabla u_{1}+\nabla_{y} \widehat{u}_{1}\right)\left(\nabla \varphi+\nabla_{y} \Phi_{1}\right) \mathrm{d} x \mathrm{~d} y+\int_{\Omega \times Y_{2}} A(y) \nabla_{y} \widehat{u}_{2} \nabla_{y} \Phi_{2} \mathrm{~d} x \mathrm{~d} y+ \\
\int_{\Omega \times \Gamma} h(y)\left(u_{1}-\widehat{u}_{2}\right)\left(\varphi-\Phi_{2}\right) \mathrm{d} x \mathrm{~d} \sigma_{y}=\int_{\Omega \times Y_{1}} f(x) \varphi(x) \mathrm{d} x \mathrm{~d} y+ \\
\int_{\Omega \times Y_{2}} f(x) \Phi_{2}(x, y) \mathrm{d} x \mathrm{~d} y+\int_{\Omega \times \Gamma} g(y) \Phi_{1}(x, y) \mathrm{d} x \mathrm{~d} \sigma_{y},
\end{gathered}
$$

for all $\varphi \in H_{0}^{1}(\Omega), \Phi_{1} \in L^{2}\left(\Omega, H_{p e r}^{1}\left(Y_{1}\right)\right), \Phi_{2} \in L^{2}\left(\Omega, H^{1}\left(Y_{2}\right)\right)$.

Proof. To obtain the problem (3.22), we pass to the limit in the unfolded form of the variational formulation (2.6) with the test functions (3.3), which satisfy (3.4)-(3.7). The only difference with respect to the proof of Theorem 3.2 is the passage to the limit in the term involving the function $G^{\varepsilon}$. More precisely, we have now:

$$
\begin{gathered}
\frac{1}{\varepsilon} \int_{\Omega \times \Gamma} \mathcal{T}_{b}^{\varepsilon}\left(G^{\varepsilon}\right) \mathcal{T}_{b}^{\varepsilon}\left(v_{1}\right) \mathrm{d} \sigma_{x}=\frac{1}{\varepsilon} \int_{\Omega \times \Gamma} \mathcal{T}_{b}^{\varepsilon}\left(g\left(\frac{x}{\varepsilon}\right)\right) \mathcal{T}_{b}^{\varepsilon}\left(\varphi(x)+\varepsilon \omega_{1}(x) \psi_{1}\left(\frac{x}{\varepsilon}\right)\right) \mathrm{d} \sigma_{x}= \\
\frac{1}{\varepsilon} \int_{\Omega \times \Gamma} g(y) \mathcal{T}_{b}^{\varepsilon}(\varphi)(x, y) \mathrm{d} x \mathrm{~d} \sigma_{y}+\int_{\Omega \times \Gamma} g(y) \mathcal{T}_{b}^{\varepsilon}\left(\omega_{1}\right)(x, y) \mathcal{T}_{b}^{\varepsilon}\left(\psi_{1}\right)(x, y) \mathrm{d} x \mathrm{~d} \sigma_{y} \rightarrow \\
\int_{\Omega \times \Gamma} g(y) \omega_{1}(x) \psi_{1}(y) \mathrm{d} x \mathrm{~d} \sigma_{y} .
\end{gathered}
$$

from the fact that $\mathcal{M}_{\Gamma}(g)=0$. By using the density of $\mathcal{D}(\Omega) \otimes H_{\text {per }}^{1}\left(Y_{1}\right)$ in $L^{2}\left(\Omega, H_{\text {per }}^{1}\left(Y_{1}\right)\right)$ and of $\mathcal{D}(\Omega) \otimes H^{1}\left(Y_{2}\right)$ in $L^{2}\left(\Omega, H^{1}\left(Y_{2}\right)\right)$, we are led to the unfolded limit problem (3.22).

Due to the uniqueness of $\left(u_{1}, \widehat{u}_{1}, \widehat{u}_{2}\right) \in \mathcal{V}$, which can be proven by the Lax-Milgram theorem, all the above convergences hold true for the whole sequence and our theorem is proven. 
Remark 3.9 Let us notice that the term $\int_{\Omega \times \Gamma} g(y) \Phi_{1}(x, y) \mathrm{d} x \mathrm{~d} \sigma_{y}$ in (3.22) constitutes the main difference with respect to the unfolded equation (3.2), where the term involving $g$ is a nonzero constant, appearing explicitly in the right-hand side of the homogenized equation (3.8). This is not the case here, since this term involves now explicitly both variables $x$ and $y$. Our task now is to understand the contribution in the homogenized problem of this non standard term generated by the discontinuity of the flux in the microscopic problem. As we shall see in Theorem 3.10, apart from the cell problems (3.12) and (3.13), an additional non homogeneous Neumann cell problem needs to be introduced.

Theorem 3.10 The unique solution $u^{\varepsilon}=\left(u_{1}^{\varepsilon}, u_{2}^{\varepsilon}\right)$ of the variational problem (2.6) converges, in the sense of (3.1), to $\left(u_{1}, \widehat{u}_{1}, \widehat{u}_{2}\right) \in \mathcal{V}$, where $u_{1}$ is the unique solution of the homogenized problem

$$
\left\{\begin{array}{l}
-\operatorname{div}\left(A^{h o m} \nabla u_{1}(x)\right)=f(x) \quad \text { in } \Omega \\
u_{1}=0 \quad \text { on } \partial \Omega
\end{array}\right.
$$

and

$$
\begin{gathered}
\widehat{u}_{1}(x, y)=-\sum_{j=1}^{N} \frac{\partial u_{1}}{\partial x_{j}}(x) \chi_{1}^{j}(y)+\eta(y), \\
\widehat{u}_{2}(x, y)=u_{1}(x)+f(x) \chi_{2}(y) .
\end{gathered}
$$

Here, $A^{\text {hom }}$ is the homogenized matrix whose entries are given by (3.11) and the functions $\chi_{1}^{j}$ and $\chi_{2}$ are defined by (3.12) and (3.13). The $Y$-periodic function $\eta$ is the unique solution of the following non homogeneous Neumann cell problem:

$$
\left\{\begin{array}{l}
-\operatorname{div}_{y}\left(A(y) \nabla_{y} \eta\right)=0 \quad \text { in } Y_{1}, \\
A(y) \nabla_{y} \eta \cdot n=-g(y) \quad \text { on } \Gamma, \\
\mathcal{M}_{\Gamma}(\eta)=0 .
\end{array}\right.
$$

Convergences (3.14) and (3.15) still hold true, with $u_{1}$ solution of (3.23).

Proof. By taking $\varphi=0$ in the unfolded limit problem (3.22), we obtain:

$$
\begin{gathered}
\int_{\Omega \times Y_{1}} A(y)\left(\nabla u_{1}+\nabla_{y} \widehat{u}_{1}\right) \nabla_{y} \Phi_{1} \mathrm{~d} x \mathrm{~d} y+\int_{\Omega \times Y_{2}} A(y) \nabla_{y} \widehat{u}_{2} \nabla_{y} \Phi_{2} \mathrm{~d} x \mathrm{~d} y- \\
\int_{\Omega \times \Gamma} h(y)\left(u_{1}-\widehat{u}_{2}\right) \Phi_{2} \mathrm{~d} x \mathrm{~d} \sigma_{y}= \\
\int_{\Omega \times Y_{2}} f(x) \Phi_{2}(x, y) \mathrm{d} x \mathrm{~d} y+\int_{\Omega \times \Gamma} g(y) \Phi_{1}(x, y) \mathrm{d} x \mathrm{~d} \sigma_{y} .
\end{gathered}
$$

By choosing $\Phi_{1}=0$ in (3.26), we have

$$
\int_{\Omega \times Y_{2}} A(y) \nabla_{y} \widehat{u}_{2} \nabla_{y} \Phi_{2} \mathrm{~d} x \mathrm{~d} y-\int_{\Omega \times \Gamma} h(y)\left(u_{1}-\widehat{u}_{2}\right) \Phi_{2} \mathrm{~d} x \mathrm{~d} \sigma_{y}=\int_{\Omega \times Y_{2}} f(x) \Phi_{2} \mathrm{~d} x \mathrm{~d} y .
$$


By taking now suitable test functions $\Phi_{2}$, we obtain

$$
-\operatorname{div}_{y}\left(A(y) \nabla_{y} \widehat{u}_{2}\right)=f \quad \text { in } \Omega \times Y_{2}
$$

and

$$
A(y) \nabla_{y} \widehat{u}_{2} \cdot n=h(y)\left(u_{1}-\widehat{u}_{2}\right) \quad \text { on } \Omega \times \Gamma .
$$

We then find the functions $\widehat{u}_{2}$ and $\chi_{2}$ exactly like in the proof of Theorem 3.3.

Now, let us take $\Phi_{2}=0$ in (3.26). We obtain

$$
\int_{\Omega \times Y_{1}} A(y)\left(\nabla u_{1}+\nabla_{y} \widehat{u}_{1}\right) \nabla_{y} \Phi_{1} \mathrm{~d} x \mathrm{~d} y=\int_{\Omega \times \Gamma} g(y) \Phi_{1} \mathrm{~d} x \mathrm{~d} \sigma_{y} .
$$

By taking suitable test functions $\Phi_{1}$, we obtain

$$
\begin{gathered}
-\operatorname{div}_{y}\left(A(y) \nabla_{y} \widehat{u}_{1}\right)=\operatorname{div}_{y}\left(A(y) \nabla u_{1}\right) \quad \text { in } \Omega \times Y_{1}, \\
A(y)\left(\nabla_{x} u_{1}+\nabla_{y} \widehat{u}_{1}\right) \cdot n=-g(y) \quad \text { on } \Omega \times \Gamma .
\end{gathered}
$$

We remark that (3.27) and (3.29) imply that we also have a discontinuity type condition:

$$
A(y)\left(\nabla_{x} u_{1}+\nabla_{y} \widehat{u}_{1}\right) \cdot n-A(y) \nabla_{y} \widehat{u}_{2} \cdot n=-h(y)\left(u_{1}-\widehat{u}_{2}\right)-g(y) \quad \text { on } \Omega \times \Gamma .
$$

The presence of the function $g$ in relation (3.29) suggests us to search $\widehat{u}_{1}$ in the following non standard form:

$$
\widehat{u}_{1}(x, y)=-\sum_{j=1}^{N} \frac{\partial u_{1}}{\partial x_{j}}(x) \chi_{1}^{j}(y)+\eta(y),
$$

where the functions $\chi_{1}^{j}$ are defined by (3.12) and the function $\eta$ remains to be found. To this end, we replace $\widehat{u}_{1}$ given by (3.30) in (3.28)-(3.29). We obtain:

$$
\left\{\begin{array}{l}
-\operatorname{div}_{y}\left(A(y)\left(-\nabla_{x} u_{1} \nabla_{y} \chi_{1}+\nabla_{y} \eta(y)\right)=\operatorname{div}_{y}\left(A(y) \nabla_{x} u_{1}\right) \quad \text { in } \Omega \times Y_{1},\right. \\
A(y)\left(\nabla_{x} u_{1}-\nabla_{x} u_{1} \nabla_{y} \chi_{1}+\nabla_{y} \eta\right) \cdot n=-g(y) \quad \text { on } \Omega \times \Gamma, \\
\mathcal{M}_{\Gamma}(\eta)=0 .
\end{array}\right.
$$

By using (3.12), we deduce that the scalar function $\eta$ is the unique $Y$-periodic solution of the cell problem

$$
\begin{cases}-\operatorname{div}_{y}\left(A(y) \nabla_{y} \eta\right)=0 & \text { in } Y_{1} \\ A(y) \nabla_{y} \eta \cdot n=-g(y) & \text { on } \Gamma \\ \mathcal{M}_{\Gamma}(\eta)=0 & \end{cases}
$$

We observe that (3.32) is a non homogeneous Neumann problem. The compatibility condition

$$
\int_{\Gamma} \eta(y) \mathrm{d} y=0
$$


is satisfied, thanks to the hypothesis (2.3) imposed on the function $g$.

By choosing now $\Phi_{1}=\Phi_{2}=0$ in (3.22), we get:

$$
\begin{gathered}
\int_{\Omega \times Y_{1}} A(y)\left(\nabla u_{1}+\nabla_{y} \widehat{u}_{1}\right) \nabla \varphi \mathrm{d} x \mathrm{~d} y+\int_{\Omega \times \Gamma} h(y)\left(u_{1}-\widehat{u}_{2}\right) \varphi \mathrm{d} x \mathrm{~d} \sigma_{y}= \\
\int_{\Omega \times Y_{1}} f(x) \varphi(x) \mathrm{d} x \mathrm{~d} y .
\end{gathered}
$$

Also, since

$$
u_{1}(x)-\widehat{u}_{2}(x, y)=-f_{2}(x) \chi_{2}(y) \quad \text { in } \Omega \times Y_{2},
$$

we have, as in the proof of Theorem 3.3, the equality

$$
\int_{\Omega \times \Gamma} h(y)\left(u_{1}-\widehat{u}_{2}\right) \varphi \mathrm{d} x \mathrm{~d} \sigma_{y}=-\left|Y_{2}\right| \int_{\Omega} f(x) \varphi(x) \mathrm{d} x
$$

and relation (3.33) then becomes:

$$
\int_{\Omega \times Y_{1}} A(y)\left(\nabla u_{1}+\nabla_{y} \widehat{u}_{1}\right) \nabla \varphi \mathrm{d} x \mathrm{~d} y=\int_{\Omega} f(x) \varphi(x) \mathrm{d} x .
$$

We integrate this last equality by parts with respect to $x$ and, using (3.30) and the definition (3.11) of the matrix $A^{\text {hom }}$, we obtain

$$
-\operatorname{div}_{x}\left(A^{\mathrm{hom}} \nabla u_{1}\right)=f+\operatorname{div}_{x}\left(\int_{Y_{1}} A(y) \nabla \eta(y) \mathrm{d} y\right) \quad \text { in } \Omega,
$$

which leads immediately to the homogenized problem (3.23). We notice that this problem does not involve the function $g$. Nevertheless, the influence of the flux jump $g$ appears in the corrector function $\widehat{u}_{1}$, via the cell problem (3.25).

Remark 3.11 The above results can be generalized to the case in which $A^{\varepsilon}$ is a sequence of matrices in $\mathcal{M}(\lambda, \mu, \Omega)$ such that

$$
\mathcal{T}_{\alpha}^{\varepsilon}\left(A^{\varepsilon}\right) \rightarrow \text { A strongly in } L^{1}(\Omega \times Y)
$$

for some matrix $A=A(x, y)$ in $\mathcal{M}(\lambda, \mu, \Omega \times Y)$. The heterogeneity of the medium described by such a matrix generates different effects in our limit problems (3.2) and (3.22), respectively. In both situations, due to the fact that the correctors $\chi_{1}^{j}$ depend also on the variable $x$, the new homogenized matrix $A_{x}^{\text {hom }}$ is no longer constant, but it depends on $x$. A more interesting effect occurs in the second case. As proven in Theorem 3.10, if the matrix A depends only on the variable $y$, the function $\eta$ is independent of $x$ and there is no contribution of the term containing $g$ in the decoupled form of the limit problem. So, the limit equation is the same as that corresponding to the case with no jump of the flux in the microscopic problem. Now, the 
dependence of $A$ on $x$ prevents this phenomenon to occur, and, as a consequence, the function $g$ gives an explicit contribution in the homogenized problem, which becomes

$$
-\operatorname{div}_{x}\left(A_{x}^{h o m} \nabla u\right)=f+\operatorname{div}_{x}\left(\int_{Y_{1}} A(x, y) \nabla \eta(x, y) \mathrm{d} y\right) \quad \text { in } \Omega .
$$

A similar effect was observed in the homogenization of the Neumann problem in perforated domains (see [14]).

In this second case too, a corrector result similar to the one stated in Theorem 3.7 holds true. The main difference now is that the function $\eta$, solution of the cell problem (3.25), appears in the correctors of the solution $u^{\varepsilon}=\left(u_{1}^{\varepsilon}, u_{2}^{\varepsilon}\right)$ of problem (2.1), as well.

Theorem 3.12 Under the assumptions of Theorem 3.8, if $u^{\varepsilon}=\left(u_{1}^{\varepsilon}, u_{2}^{\varepsilon}\right)$ is the unique solution of problem (2.1), then (3.18)-(3.21) hold true. Moreover, we have the following corrector result:

$$
\left\|\nabla u_{1}^{\varepsilon}-\nabla u_{1}+\sum_{j=1}^{N} \mathcal{U}_{1}^{\varepsilon}\left(\frac{\partial u_{1}}{\partial x_{j}}\right) \mathcal{U}_{1}^{\varepsilon}\left(\nabla_{y} \chi_{1}^{j}\right)-\mathcal{U}_{1}^{\varepsilon}\left(\nabla_{y} \eta\right)\right\|_{L^{2}\left(\Omega_{1}^{\varepsilon}\right)} \longrightarrow 0
$$

and

$$
\left\|\nabla u_{2}^{\varepsilon}-\nabla u_{1}-f(x) \mathcal{U}_{2}^{\varepsilon}\left(\nabla_{y} \chi_{2}\right)\right\|_{L^{2}\left(\Omega_{2}^{\varepsilon}\right)} \longrightarrow 0
$$

Remark 3.13 Let us point out that similar corrector results can be stated in the case in which the matrix $A$ depends both on $x$ and $y$, as in Remark 3.11.

\section{Conclusions}

Using the periodic unfolding method, the effective thermal transfer in a periodic composite material formed by two constituents, with different thermal properties, was analyzed. The main features of the considered composite material were the discontinuity of the thermal conductivity over the domain as we go from one constituent to another and the presence of an imperfect interface between the two constituents, where both the temperature and the flux exhibit jumps. The limit problem captures the influence of the jumps in the limit temperature field, in an additional source term, and in the correctors, as well.

\section{Appendix}

We briefly recall here the definitions and the main properties of the unfolding operators $\mathcal{T}_{1}^{\varepsilon}$ and $\mathcal{T}_{2}^{\varepsilon}$, introduced, for a two-component domain, by P. Donato et al. in [20] (see, also, [14], [15], [16] and [19]) and of the boundary unfolding operator $\mathcal{T}_{b}^{\varepsilon}$, introduced in [16] and [17]. The main 
particularity of these operators is that they map functions defined on the oscillating domains $\Omega_{1}^{\varepsilon}, \Omega_{2}^{\varepsilon}$ and, respectively, $\Gamma^{\varepsilon}$, into functions defined on the fixed domains $\Omega \times Y_{1}, \Omega \times Y_{2}$ and $\Omega \times \Gamma$, respectively.

For $x \in \mathbb{R}^{N}$, we denote by $[x]_{Y}$ its integer part $k \in \mathbb{Z}^{N}$, such that $x-[x]_{Y} \in Y$ and we set $\{x\}_{Y}=x-[x]_{Y}$ for $x \in \mathbb{R}^{N}$. So, for every $x \in \mathbb{R}^{N}$, we have $x=\varepsilon\left(\left[\frac{x}{\varepsilon}\right]_{Y}+\left\{\frac{x}{\varepsilon}\right\}_{Y}\right)$. For defining the above mentioned periodic unfolding operators, we consider the following sets (see [20]):

$$
\begin{gathered}
\widehat{\mathbb{Z}}_{\varepsilon}=\left\{k \in \mathbb{Z}^{N} \mid \varepsilon Y^{k} \subset \Omega\right\}, \quad \widehat{\Omega}^{\varepsilon}=\operatorname{int} \bigcup_{k \in \widehat{\mathbb{Z}}_{\varepsilon}}\left(\varepsilon \bar{Y}^{k}\right), \quad \Lambda^{\varepsilon}=\Omega \backslash \widehat{\Omega}^{\varepsilon}, \\
\widehat{\Omega}_{\alpha}^{\varepsilon}=\bigcup_{k \in \widehat{\mathbb{Z}}_{\varepsilon}}\left(\varepsilon Y_{\alpha}^{k}\right), \quad \Lambda_{\alpha}^{\varepsilon}=\Omega_{\alpha}^{\varepsilon} \backslash \widehat{\Omega}_{\alpha}^{\varepsilon}, \quad \widehat{\Gamma}^{\varepsilon}=\partial \widehat{\Omega}_{2}^{\varepsilon} .
\end{gathered}
$$

Definition 5.1 For any Lebesgue measurable function $\varphi$ on $\Omega_{\alpha}^{\varepsilon}, \alpha \in\{1,2\}$, we define the periodic unfolding operators by the formula

$$
\mathcal{T}_{\alpha}^{\varepsilon}(\varphi)(x, y)= \begin{cases}\varphi\left(\varepsilon\left[\frac{x}{\varepsilon}\right]_{Y}+\varepsilon y\right) & \text { for a.e. }(x, y) \in \widehat{\Omega}^{\varepsilon} \times Y_{\alpha} \\ 0 & \text { for a.e. }(x, y) \in \Lambda^{\varepsilon} \times Y_{\alpha}\end{cases}
$$

If $\varphi$ is a function defined in $\Omega$, for simplicity, we write $\mathcal{T}_{\alpha}^{\varepsilon}(\varphi)$ instead of $\mathcal{T}_{\alpha}^{\varepsilon}\left(\left.\varphi\right|_{\Omega_{\alpha}^{\varepsilon}}\right)$.

For any function $\varphi$ which is Lebesgue-measurable on $\Gamma^{\varepsilon}$, the periodic boundary unfolding operator $\mathcal{T}_{b}^{\varepsilon}$ is defined by

$$
\mathcal{T}_{b}^{\varepsilon}(\varphi)(x, y)= \begin{cases}\varphi\left(\varepsilon\left[\frac{x}{\varepsilon}\right]_{Y}+\varepsilon y\right) & \text { for a.e. }(x, y) \in \widehat{\Omega}^{\varepsilon} \times \Gamma \\ 0 & \text { for a.e. }(x, y) \in \Lambda^{\varepsilon} \times \Gamma\end{cases}
$$

Remark 5.2 We notice that if $\varphi \in H^{1}\left(\Omega_{\alpha}^{\varepsilon}\right)$, then $\mathcal{T}_{b}^{\varepsilon}(\varphi)=\left.\mathcal{T}_{\alpha}^{\varepsilon}(\varphi)\right|_{\widehat{\Omega}^{\varepsilon} \times \Gamma}$.

We give now a few useful properties of these operators (see, e.g., [14], [19] and [20]).

Proposition 5.3 For $p \in[1, \infty)$ and $\alpha \in\{1,2\}$, the operators $\mathcal{T}_{\alpha}^{\varepsilon}$ are linear and continuous from $L^{p}\left(\Omega_{\alpha}^{\varepsilon}\right)$ to $L^{p}\left(\Omega \times Y_{\alpha}\right)$ and

(i) if $\varphi$ and $\psi$ are two Lebesgue measurable functions on $\Omega_{\alpha}^{\varepsilon}$, one has $\mathcal{T}_{\alpha}^{\varepsilon}(\varphi \psi)=\mathcal{T}_{\alpha}^{\varepsilon}(\varphi) \mathcal{T}_{\alpha}^{\varepsilon}(\psi)$;

(ii) for every $\varphi \in L^{1}\left(\Omega_{\alpha}^{\varepsilon}\right)$, one has

$$
\frac{1}{|Y|} \int_{\Omega \times Y_{\alpha}} \mathcal{T}_{\alpha}^{\varepsilon}(\varphi)(x, y) \mathrm{d} x \mathrm{~d} y=\int_{\widehat{\Omega}_{\alpha}^{\varepsilon}} \varphi(x) \mathrm{d} x=\int_{\Omega_{\alpha}^{\varepsilon}} \varphi(x) \mathrm{d} x-\int_{\Lambda_{\varepsilon}} \varphi(x) \mathrm{d} x ;
$$

(iii) if $\left\{\varphi^{\varepsilon}\right\}_{\varepsilon} \subset L^{p}(\Omega)$ is a sequence such that $\varphi^{\varepsilon} \longrightarrow \varphi$ strongly in $L^{p}(\Omega)$, then $\mathcal{T}_{\alpha}^{\varepsilon}\left(\varphi^{\varepsilon}\right) \longrightarrow \varphi$ strongly in $L^{p}\left(\Omega \times Y_{\alpha}\right)$;

(iv) if $\varphi \in L^{p}\left(Y_{\alpha}\right)$ is $Y$-periodic and $\varphi^{\varepsilon}(x)=\varphi(x / \varepsilon)$, then $\mathcal{T}_{\alpha}^{\varepsilon}\left(\varphi^{\varepsilon}\right) \longrightarrow \varphi$ strongly in $L^{p}\left(\Omega \times Y_{\alpha}\right) ;$ 
(v) if $\varphi \in W^{1, p}\left(\Omega_{\alpha}^{\varepsilon}\right)$, then $\nabla_{y}\left(\mathcal{T}_{\alpha}^{\varepsilon}(\varphi)\right)=\varepsilon \mathcal{T}_{\alpha}^{\varepsilon}(\nabla \varphi)$ and $\mathcal{T}_{\alpha}^{\varepsilon}(\varphi)$ belongs to $L^{2}\left(\Omega ; W^{1, p}\left(Y_{\alpha}\right)\right)$. Moreover, for every $\varphi \in L^{1}\left(\Gamma^{\varepsilon}\right)$, one has

$$
\int_{\widehat{\Gamma}^{\varepsilon}} \varphi(x) \mathrm{d} \sigma_{x}=\frac{1}{\varepsilon|Y|} \int_{\Omega \times \Gamma} \mathcal{T}_{b}^{\varepsilon}(\varphi)(x, y) \mathrm{d} x \mathrm{~d} \sigma_{y} .
$$

The following result was proven, for our geometry, in [20].

Lemma 5.4 If $u^{\varepsilon}=\left(u_{1}^{\varepsilon}, u_{2}^{\varepsilon}\right)$ is a sequence in $H^{\varepsilon}$ and $\varphi \in \mathcal{D}(\Omega)$, then, for $\varepsilon$ small enough and $\alpha \in\{1,2\}$ we have

$$
\varepsilon \int_{\Gamma^{\varepsilon}} h^{\varepsilon}\left(u_{1}^{\varepsilon}-u_{2}^{\varepsilon}\right) \varphi \mathrm{d} \sigma_{x}=\int_{\Omega \times \Gamma} h(y)\left(\mathcal{T}_{1}^{\varepsilon}\left(u_{1}^{\varepsilon}\right)-\mathcal{T}_{2}^{\varepsilon}\left(u_{2}^{\varepsilon}\right)\right) \mathcal{T}_{\alpha}^{\varepsilon}(\varphi) \mathrm{d} x \mathrm{~d} \sigma_{y} .
$$

We remind some general compactness results obtained in [22] for bounded sequences in $H^{\varepsilon}$.

Lemma 5.5 Let $u^{\varepsilon}=\left(u_{1}^{\varepsilon}, u_{2}^{\varepsilon}\right)$ be a bounded sequence in $H^{\varepsilon}$. Then, there exists a constant $C>0$, independent of $\varepsilon$, such that

$$
\begin{aligned}
& \left\|\mathcal{T}_{1}^{\varepsilon}\left(u_{1}^{\varepsilon}\right)\right\|_{L^{2}\left(\Omega \times Y_{1}\right)} \leq C, \\
& \left\|\mathcal{T}_{2}^{\varepsilon}\left(u_{2}^{\varepsilon}\right)\right\|_{L^{2}\left(\Omega \times Y_{2}\right)} \leq C, \\
& \left\|\mathcal{T}_{1}^{\varepsilon}\left(\nabla u_{1}^{\varepsilon}\right)\right\|_{L^{2}\left(\Omega \times Y_{1}\right)} \leq C, \\
& \varepsilon\left\|\mathcal{T}_{2}^{\varepsilon}\left(\nabla u_{2}^{\varepsilon}\right)\right\|_{L^{2}\left(\Omega \times Y_{2}\right)} \leq C, \\
& \left\|\mathcal{T}_{2}^{\varepsilon}\left(u_{1}^{\varepsilon}\right)-\mathcal{T}_{1}^{\varepsilon}\left(u_{2}^{\varepsilon}\right)\right\|_{L^{2}(\Omega \times \Gamma)} \leq C .
\end{aligned}
$$

Proposition 5.6 Let $u^{\varepsilon}=\left(u_{1}^{\varepsilon}, u_{2}^{\varepsilon}\right)$ be a bounded sequence in $H^{\varepsilon}$. Then, up to a subsequence, still denoted by $\varepsilon$, there exist $u_{1} \in H_{0}^{1}(\Omega), \widehat{u}_{1} \in L^{2}\left(\Omega, H_{\text {per }}^{1}\left(Y_{1}\right)\right)$ and $\widehat{u}_{2} \in L^{2}\left(\Omega, H^{1}\left(Y_{2}\right)\right)$ such that

$$
\begin{aligned}
& \mathcal{T}_{1}^{\varepsilon}\left(u_{1}^{\varepsilon}\right) \longrightarrow u_{1} \text { strongly in } L^{2}\left(\Omega, H^{1}\left(Y_{1}\right)\right), \\
& \mathcal{T}_{1}^{\varepsilon}\left(\nabla u_{1}^{\varepsilon}\right) \rightarrow \nabla u_{1}+\nabla_{y} \widehat{u}_{1} \text { weakly in } L^{2}\left(\Omega \times Y_{1}\right), \\
& \mathcal{T}_{2}^{\varepsilon}\left(u_{2}^{\varepsilon}\right) \rightarrow \widehat{u}_{2} \text { weakly in } L^{2}\left(\Omega, H^{1}\left(Y_{2}\right)\right), \\
& \varepsilon \mathcal{T}_{2}^{\varepsilon}\left(\nabla u_{2}^{\varepsilon}\right) \rightarrow \nabla_{y} \widehat{u}_{2} \text { weakly in } L^{2}\left(\Omega \times Y_{2}\right), \\
& \widetilde{u}_{1}^{\varepsilon} \rightarrow \frac{\left|Y_{1}\right|}{|Y|} u_{1} \text { weakly in } L^{2}(\Omega), \\
& \widetilde{u}_{2}^{\varepsilon} \rightarrow \frac{1}{|Y|} \int_{Y_{2}} \widehat{u}_{2}(x, y) \mathrm{d} y \quad \text { weakly in } L^{2}(\Omega) .
\end{aligned}
$$

where $\mathcal{M}_{\Gamma}\left(\widehat{u}_{1}\right)=0$ for almost every $x \in \Omega$ and $\tilde{x}$ denotes the extension by zero of a function to the whole of the domain $\Omega$. 
Finally, we give for $\alpha \in\{1,2\}$ the definition of the adjoints $\mathcal{U}_{\alpha}$ of the unfolding operators and we state some useful properties for them (see [14] and [20]).

Definition 5.7 For $p \in[1, \infty]$, the averaging operators $\mathcal{U}_{\alpha}^{\varepsilon}: L^{p}\left(\Omega \times Y_{\alpha}\right) \rightarrow L^{p}\left(\Omega_{\alpha}^{\varepsilon}\right)$, are given by

$$
\mathcal{U}_{\alpha}^{\varepsilon}(\phi)(x)= \begin{cases}\frac{1}{|Y|} \int_{Y} \phi\left(\varepsilon\left[\frac{x}{\varepsilon}\right]_{Y}+\varepsilon z,\left\{\frac{x}{\varepsilon}\right\}_{Y}\right) \mathrm{d} z & \text { for a.e. } x \in \widehat{\Omega}_{\alpha}^{\varepsilon} \\ 0 & \text { for a.e. } x \in \Lambda_{\alpha}^{\varepsilon}\end{cases}
$$

It is not difficult to see that these averaging operators are almost left-inverses of the corresponding unfolding operators $\mathcal{T}_{\alpha}^{\varepsilon}$, i.e., for any $\varphi \in L^{p}\left(\Omega_{\alpha}^{\varepsilon}\right)$, we have

$$
\mathcal{U}_{\alpha}^{\varepsilon}\left(\mathcal{T}_{\alpha}^{\varepsilon}(\varphi)\right)(x)= \begin{cases}\varphi(x) & \text { for a.e. } x \in \widehat{\Omega}_{\alpha}^{\varepsilon} \\ 0 & \text { for a.e. } x \in \Lambda_{\alpha}^{\varepsilon}\end{cases}
$$

Proposition 5.8 For $p \in[1, \infty)$, the operators $\mathcal{U}_{\alpha}^{\varepsilon}$ are linear and continuous from $L^{p}\left(\Omega \times Y_{\alpha}\right)$ to $L^{p}\left(\Omega_{\alpha}^{\varepsilon}\right)$ and

(i) $\left\|\mathcal{U}_{\alpha}^{\varepsilon}(\phi)-\phi\right\|_{L^{p}\left(\Omega_{\alpha}^{\varepsilon}\right)} \rightarrow 0$ for every $\phi \in L^{p}(\Omega)$;

(ii) if $\varphi_{\varepsilon} \in L^{p}\left(\Omega_{\alpha}^{\varepsilon}\right)$, then the following statements are equivalent:

- $\mathcal{T}_{\alpha}^{\varepsilon}\left(\varphi_{\varepsilon}\right) \rightarrow \widehat{\varphi}$ strongly in $L^{p}\left(\Omega \times Y_{\alpha}\right)$ and $\int_{\Lambda_{\alpha}^{\varepsilon}}\left|\varphi_{\varepsilon}\right|^{p} \mathrm{~d} x \rightarrow 0$;

- $\left\|\varphi_{\varepsilon}-\mathcal{U}_{\alpha}^{\varepsilon}(\widehat{\varphi})\right\|_{L^{p}\left(\Omega_{\alpha}^{\varepsilon}\right)} \rightarrow 0$.

Acknowledgements. Both authors acknowledge the support from the Laboratoire Européen Associé CNRS Franco-Roumain "Math-Mode".

\section{References}

[1] A. Ainouz, Homogenized double porosity models for poro-elastic media with interfacial flow barrier, Math. Bohem. 136, 357365, 2011.

[2] A. Ainouz, Homogenization of a dual-permeability problem in two-component media with imperfect contact, Applications of Mathematics 2, 185-196, 2015.

[3] G. Allaire, Homogenization and two-scale convergence, SIAM J. Math. Anal. 23, 1482-1518, 1992.

[4] M. Amar, D. Andreucci, R. Gianni, Evolution and memory effects in the homogenization limit for electrical conduction in biological tissues, Math. Model. Methods Appl. Sci. 14, 1261-1295, 2004. 
[5] M. Amar, D. Andreucci, P. Bisegna, R. Gianni, A hierarchy of models for the electrical conduction in biological tissues via two-scale convergence: The nonlinear case, Differ. Integral Equations, 26 (9-10), 885-912, 2013.

[6] T. Arbogast, J. Douglas Jr., U. Hornung, Derivation of the double porosity model of single phase flow via homogenization theory, SIAM J. Math. Anal., 21, pp. 823- 836, 1990.

[7] J. L. Auriault, C. Boutin, C. Geindreau, Homogenization of Coupled Phenomena in Heterogenous Media, Wiley, 2010.

[8] J. L. Auriault, H. Ene, Macroscopic modelling of heat transfer in composites with interfacial thermal barrier, Int. J. of Heat and Mass Transfer, 37 (18), 2885-2892, 1994.

[9] G. I. Barenblatt, I.P. Zheltov, I.N. Kochina, Basic concepts in the theory of seepage of homogeneous liquids in fissured rocks (strata), Prikl. Mat. Mekh., 24, 852-864, 1960, J. Appl. Math. Mech., 24, 1286-1303, 1960.

[10] Y. Benveniste, T. Miloh, Imperfect soft and stiff interfaces in two-dimensional elasticity, Mech. Mater., 33, 309-324, 2001.

[11] D. Brinkman, K. Fellner, P. Markowich, M. T. Wolfram, A Drift-Diffusion- Reaction Model for Excitonic Photovoltaic Bilayers: Asymptotic Analysis and a 2-D HDG Finite Element Scheme, Math. Models Methods Appl. Sci., 23, 839-872, 2013.

[12] R. Bunoiu, C. Timofte, Homogenization of a Thermal Problem with Flux Jump, Networks and Heterogeneous Media, to appear; https://hal.inria.fr/hal-01272936.

[13] R. Bunoiu, C. Timofte, On the Homogenization of a Diffusion Problem with Flux Jump, in preparation.

[14] D. Cioranescu, A. Damlamian, P. Donato, G. Griso, R. Zaki, The periodic unfolding method in domains with holes, SIAM J. Math. Anal. 44, 718-760, 2012.

[15] D. Cioranescu, A. Damlamian, G. Griso, The periodic unfolding method in homogenization, SIAM J. Math. Anal., 40 (4), 1585-1620, 2008.

[16] D. Cioranescu, P. Donato, R. Zaki, The periodic unfolding method in perforated domains, Portugaliae Math. 63 (4), 467-496, 2006.

[17] D. Cioranescu, P. Donato, R. Zaki, Asymptotic behavior of elliptic problems in perforated domains with nonlinear boundary conditions, Asymptot. Anal. 53, 209-235, 2007. 
[18] I. Chourabi and P. Donato, Homogenization and correctors of a class of elliptic problems in perforated domains, Asymptot. Anal., 92, 1-43, DOI 10.3233/ASY-151288, 2015.

[19] P. Donato, K. H. Le Nguyen, Homogenization of diffusion problems with a nonlinear interfacial resistance, Nonlinear Differ. Equ. Appl., DOI 10.1007/s00030-015-0325-2, 2015.

[20] P. Donato, K. H. Le Nguyen, R. Tardieu, The periodic unfolding method for a class of imperfect transmission problems, J. Math. Sci. (N. Y.), 6 (176), 891-927, 2011.

[21] P. Donato, S. Monsurrò, Homogenization of two heat conductors with an interfacial contact resistance, Analysis and Applications, 2 (3), 247-273, 2004.

[22] P. Donato, I. Țenţea, Homogenization of an elastic double-porosity medium with imperfect interface via the periodic unfolding method, Boundary Value Problems, 2013:265, 2013.

[23] H. I. Ene, D. Poliševski, Model of diffusion in partially fissured media, Z. Angew. Math. Phys., 6 (53), 1052-1059, 2002.

[24] H. I. Ene, C. Timofte, Microstructure models for composites with imperfect interface via the periodic unfolding method, Asymptot. Anal., 89 (1-2), 111-122, 2014.

[25] H. I. Ene, C. Timofte, I. Ţ̧enţea, Homogenization of a thermoelasticity model for a composite with imperfect interface, Bull. Math. Soc. Sci. Math. Roumanie, 58 (106), 2, 147-160, 2015.

[26] H. I. Ene, C. Timofte, Homogenization results for a dynamic coupled thermoelasticity problem, Romanian Reports in Physics, in press, 2016.

[27] K. Fellner, V. Kovtunenko, A discontinuous Poisson-Boltzmann equation with interfacial transfer: homogenisation and residual error estimate, preprint, 2014.

[28] I. Gruais, D. Polisevski, Heat transfer models for two-component media with interfacial jump, Applicable Analysis, DOI: 10.1080/00036811.2015.1124421, 2015.

[29] Z. Hashin, Thin interphase-imperfect interface in elasticity with application to coated fiber composites, Journal of the Mechanics and Physics of Solids 50, 2509-2537, 2002.

[30] H. K. Hummel, Homogenization for heat transfer in polycrystals with interfacial resistances, Appl. Anal., 75, 403-424, 2000.

[31] E. R. Ijioma, A. Muntean, T. Ogawa, Pattern formation in reverse smouldering combustion: a homogenization approach, Combustion Theory and Modelling, 17(2), 185-223, 2013. 
[32] K. H. Le Nguyen, Homogenization of heat transfer process in composite materials, JEPE, 1, 175-188, 2015.

[33] S. Monsurrò, Homogenization of a two-component composite with interfacial thermal barrier, Adv. Math. Sci. Appl., 13 (1), 43-63, 2003.

[34] D. Polisevski, R. Schiltz-Bunoiu, A. Stanescu, Homogenization cases of heat transfer in structures with interfacial barriers, Bulletin Mathématique de la Société des Sciences Mathématiques de Roumanie, 58 (106), 4, 463-473, 2015.

[35] D. Polisevski, R. Schiltz-Bunoiu, Heat conduction through a first-order jump interface, New Trends in Continuum Mechanics (M. Mihailescu-Suliciu ed.), Theta Series in Advanced Mathematics, 225-230, 2005.

[36] D. Polisevski, R. Schiltz-Bunoiu, Diffusion in an intermediate model of fractured porous media, Bulletin Scientifique, Mathématiques et Informatique, 10, 99-106, 2004.

[37] L. I. Rubinstein, On the problem of the process of propagation of heat in heterogeneous media, Izv. Akad. Nauk SSSR Ser. Geogr. 1, 1948.

[38] R. E. Showalter, Microstructure Models of Porous Media, Homogenization and Porous Media, Springer, 183-202, 1997.

[39] C. Timofte, Multiscale analysis of diffusion processes in composite media, Computers and Mathematics with Applications, 9 (66), 1573-1580, 2013.

[40] C. Timofte, Multiscale modeling of heat transfer in composite materials, Romanian Journal of Physics, 58 (9-10), 1418-1427, 2013.

[41] C. Timofte, Multiscale analysis in nonlinear thermal diffusion problems in composite structures, Cent. Eur. J. Phys., 8, 555-561, 2010. 